PROCEEDINGS OF THE

AMERICAN MATHEMATICAL SOCIETY

Volume 110, Number 4, December 1990

\title{
ON NON-SELF-ADJOINT OPERATOR ALGEBRAS
}

\author{
EDWARD G. EFFROS AND ZHONG-JIN RUAN
}

(Communicated by Paul S. Muhly)

Dedicated to Richard Arens

for his enduring contributions to mathematics

\begin{abstract}
The $M$-ideals in a (not necessarily self-adjoint) unital operator algebra are the closed two sided ideals containing a contractive relative identity. The spatial tensor product of unital operator algebras need not be a minimal matricial cross-norm tensor product.
\end{abstract}

\section{INTRODUCTION}

By an operator algebra we shall mean a norm closed algebra of bounded operators on a Hilbert space. In particular, a $C^{*}$-algebra is an operator algebra which is closed under the adjoint operation. In contrast to $C^{*}$-algebra theory, the general structure theory for non-self-adjoint operator algebras has remained largely unexplored. An abstract characterization of the unital operator algebras was only recently determined in [4], and this enabled the authors to give the first proof that the quotient of an operator algebra by a closed two sided ideal is (completely isometrically isomorphic to) an operator algebra. In this note we will consider several other interesting aspects of the category of operator algebras.

It was shown in [1] and [15] that the closed two-sided ideals in a $\mathrm{C}^{*}$-algebra are determined by their position as subspaces of the underlying Banach space. Specifically they are just the $M$-ideals in that space, i.e., the subspaces which determine an $L^{\infty}$ direct sum splitting of the second dual (see [1] and [8, Theorem 1]). Approximately ten years ago Roger Smith used the Arens' product and Smith's earlier work with Joseph Ward to characterize the $M$-ideals in function algebras [14] (see [11] for some recent applications). He showed that they are the closed algebraic ideals with a bounded relative approximate identity. In $\S 2$ we use a modification of Smith's argument to prove a noncommutative version

Received by the editors February 13, 1989; presented at the AMS meeting in Muncie, Indiana on October 27, 1989.

1980 Mathematics Subject Classification (1985 Revision). Primary 46L99.

Key words and phrases. Non-self-adjoint operator algebras, $M$-ideals, tensor products.

Both authors were supported in part by NSF. 
of this result: the $M$-ideals in a unital operator algebra are the closed two-sided ideals which contain a contractive relative approximate identity.

A distinguishing feature of noncommutative analysis is the importance of the underlying matricial norm structures (see [10]). Any linear space $V$ of operators is automatically provided with matricial norms: one has distinguished norms on each of the matrix spaces $\mathbf{M}_{n}(V)$ over $V$. The operator algebraic significance of this structure is reflected in a result essentially due to Hamana [12]. A unital complete isometry of unital operator algebras, i.e., a linear map which preserves all the matrix norms and the identity, is an algebraic isomorphism. After reviewing this result in $\S 3$, we give some examples illustrating some new phenomena that one encounters in the theory of non-self-adjoint operator algebras. In particular, isometric isomorphisms need not be completely isometric, and the spatial tensor product norm on a tensor product need not be minimal among the "matricial cross-norms." The latter answers a question of Paulsen and Power [13].

\section{The AREns' Product AND $M$-IDEALS}

If $A$ is an operator algebra on a Hilbert space $H$, we say that it inital (as an operator algebra) if it contains the identity operator $I$ on $H$. More generally, we say that a normed algebra $A$ is unital if it contains a multiplicative identity $e$ satisfying $\|e\|=1$, and that $A$ has an approximate identity if it has an approximate (contractive) identity, i.e., a net $\left\{u_{\nu}\right\}_{\nu \in \Lambda}$ in $A$ such that $\left\|u_{\nu}\right\| \leq 1$, and for all $a \in A, a u_{\nu}$, and $u_{\nu} a$ converge in norm to $a$. We say that an ideal in a normed algebra has an approximate identity if there is such a net in $J$ which is an approximate identity relative to $J$.

Given a normed algebra $A$, we let $A^{*}$ and $A^{* *}$ denote the dual and second dual spaces, and we identify $A$ with a weak* dense subspace of $A^{* *}$. We define the Arens' products on $A^{* *}$ [2] as follows (our notation is slightly different from that in [7]). Given $a \in A$ and $f \in A^{*}$, we define $a f, f a \in A^{*}$ by

$$
\langle a f, b\rangle=\langle f, b a\rangle, \quad\langle f a, b\rangle=\langle f, a b\rangle \quad(b \in A) .
$$

Given $\bar{a} \in A^{* *}$, we define $\bar{a} f, f \bar{a} \in A^{*}$ by

$$
\langle\bar{a} f, b\rangle=\langle\bar{a}, f b\rangle, \quad\langle f \bar{a}, b\rangle=\langle\bar{a}, b f\rangle \quad(b \in A) .
$$

Finally, given $\bar{a}, \bar{b} \in A^{* *}$ we define the left and right Arens' products by

$$
\langle\bar{a} \lambda \bar{b}, f\rangle=\langle\bar{a}, \bar{b} f\rangle, \quad\langle\bar{a} \rho \bar{b}, f\rangle=\langle\bar{b}, f \bar{a}\rangle \quad\left(f \in A^{*}\right) .
$$

These products are more conveniently described as limits. Letting $a_{\mu}$ and $b_{\nu} \in$ $A$ converge in the weak* topology to $\bar{a}$ and $\bar{b} \in A^{* *}$, respectively, it follows from $(2.1)-(2.3)$ that

$$
\bar{a} \lambda \bar{b}=\lim _{\mu}\left(\lim _{\nu} a_{\mu} b_{\nu}\right), \quad \bar{a} \rho \bar{b}=\lim _{\nu}\left(\lim _{\mu} a_{\mu} b_{\nu}\right) .
$$

It is important to note that these are not simultaneous limits. Owing to the Bipolar Theorem, the unit ball of $A$ is dense in that of $A^{* *}$, and as a result, 
one may assume that $\left\|a_{\mu}\right\| \leq\|a\|$, and $\left\|b_{\nu}\right\| \leq\|b\|$. It follows from (2.4) that $A^{* *}$ is a Banach algebra with respect to either left or right multiplication. Given two Banach algebras $A$ and $B$ and a homomorphism $\varphi: A \rightarrow B$, the induced map $\varphi^{* *}: A^{* *} \rightarrow B^{* *}$ is again a homomorphism with respect to either $\lambda$ or $\rho$. This was proved in [7], and is an immediate consequence of (2.4). We say that $A$ is regular if the left and right products coincide. Any subalgebra $A_{0}$ of a regular algebra is again regular since the inclusion map $l: A_{0} \hookrightarrow A$ induces a weak* homeomorphic isometry $l^{* *}$ of $A_{0}^{* *}$ onto a weak* closed subspace of $A^{* *}$ preserving the left and right Arens' products. In particular, since any $\mathrm{C}^{*}$ algebra is regular (see, e.g., [5, $§ 12]$ or $[9, \S 12]$ ), we conclude the same is true for any operator algebra $A$. We shall indicate the product in $A^{* *}$ by $(\bar{a}, \bar{b}) \rightarrow \overline{a b}$.

If $V$ is a subspace of $\mathscr{B}(H)$ for some Hilbert space, then identifying $\mathbf{M}_{n}(\mathscr{B}(H))$ with $\mathscr{B}\left(H^{n}\right)$ we let $\mathbf{M}_{n}(V)$ have the relative norm in $\mathscr{B}\left(H^{n}\right)$. Given any vector space $V$ which is "matricially normed," i.e., it is provided with norms on each of the matrix spaces $\mathbf{M}_{n}(V)$, we use the pairing

$$
\mathbf{M}_{n}(V) \times \mathbf{M}_{n}\left(V^{*}\right) \rightarrow \mathbf{C} \quad(f, v) \rightarrow\langle f, v\rangle=\sum\left\langle f_{i j}, v_{i j}\right\rangle,
$$

to identify $\mathbf{M}_{n}\left(V^{*}\right)$ and $\mathbf{M}_{n}(V)^{*}$, and thus to give $\mathbf{M}_{n}\left(V^{*}\right)$ a norm. Using these conventions, we have that if $A$ is an operator algebra, then both $A^{*}$ and $A^{* *}$ are matricially normed. $\mathbf{M}_{n}(A)$ and $\mathbf{M}_{n}\left(A^{* *}\right)$ are algebras under the multiplications determined by matrix multiplication and the corresponding products on $A$ and $A^{* *}$, respectively. If $A$ has an identity $e$, then $e$ is also an identity for $A^{* *}$. Identifying $\mathbf{M}_{n}\left(A^{* *}\right)$ with $\mathbf{M}_{n}(A)^{* *}$, the matrix product on the former is mapped into the Arens' product on the latter, since given

$$
\bar{a}=[\bar{a}(i, j)], \quad \bar{b}=[\bar{b}(i j)] \in \mathbf{M}_{n}\left(A^{* *}\right),
$$

and nets $a_{\mu}, b_{\nu} \in A$ converging to $\bar{a}$ and $\bar{b}$, respectively, we have

$$
\begin{aligned}
(\bar{a} \bar{b})(i, j) & =\lim _{\mu} \lim _{\nu}\left(a_{\mu} b_{\nu}\right)(i, j) \\
& =\lim _{\mu} \lim _{\nu} \sum_{k} a_{\mu}(i, k) b_{\nu}(k, j) \\
& =\sum_{k} \bar{a}(i, k) \bar{b}(k, j) .
\end{aligned}
$$

From the above considerations we conclude:

Theorem 2.1. Suppose that $A \subseteq \mathscr{B}(H)$ is an operator algebra (resp., unital operator algebra). Then the left and right Arens' products on the operator space $A^{* *}$ coincide, and the resulting algebra is completely isometric to a $\sigma$-weakly closed operator algebra (resp., unital operator algebra).

The fact that the second dual of a unital operator algebra is again a unital operator algebra also follows from the abstract characterization for such systems given in [4]. That the two products agree is a consequence of Hamana's theory (see Theorem 3.1). This approach does not, however, enable one to show that 
the second dual is equivalent to a $\sigma$-weakly closed operator algebra. In contrast to von Neumann algebra theory, it is not known if dual non-self-adjoint operator algebras can be realized in that manner.

Theorem 2.2. Suppose that $A \subseteq \mathscr{B}(H)$ is a unital operator algebra. Then a linear subspace $J \subseteq A$ is an $M$-ideal if and only if it is a closed two-sided ideal with an approximate identity.

Proof. Let us suppose that $J \subseteq A$ is an $M$-ideal. Then $\bar{J}$ is an $M$-summand in $A^{* *}$, i.e., we have a unique projection $P: A^{* *} \rightarrow A^{* *}$ for which $\bar{J}=P\left(A^{* *}\right)$, and

$$
\|P \bar{a}+(I-P) \bar{a}\|=\max \{\|P \bar{a}\|,\|(I-P) \bar{a}\|\}
$$

for all $\bar{a} \in A^{* *}$. To show that $J$ is an ideal in $A$, it suffices to prove that $\bar{J}$ is an ideal in $A^{* *}$, since $J=\bar{J} \cap A(J$ is weakly closed in $A)$.

We let $J_{1}=\bar{J}$, and $J_{2}=(I-P)\left(A^{* *}\right)$. From [15] we have $J_{1}$ and $J_{2}$ are closed subalgebras of $A^{* *}$ and that $z=P(I)$ is a hermitian idempotent of the Banach algebra $A^{* *}$ such that

$$
\begin{aligned}
& z A^{* *} z \subseteq J_{1}, \quad(I-z) A^{* *}(I-z) \subseteq J_{2}, \\
& z J_{2} z=0, \quad(I-z) J_{1}(I-z)=0 .
\end{aligned}
$$

Since $A^{* *}$ is an operator algebra (Theorem 2.1), $z$ is selfadjoint, i.e., it is a projection.

We claim that $z$ is central in $A^{* *}$. Since the latter is the sum of $J_{1}$ and $J_{2}$, it suffices to show that if $\bar{a} \in J_{k}(k=1,2)$, then $z \bar{a}(I-z)=(I-z) \bar{a} z=0$. Let us suppose that $\bar{a} \in J_{1},\|\bar{a}\| \leq 1$ and that $z \bar{a}(I-z) \neq 0$, so that in particular, $z \neq 0,1$. Since $z \bar{a}(I-z)=z \bar{a}-z \bar{a} z \in J_{1}$ (the latter is a subalgebra), and $I-z$ is a nonzero projection in $J_{2}$, we have that

$$
\|z \bar{a}(I-z)+(I-z)\|=\max \{\|z \bar{a}(I-z)\|,\|I-z\|\}=1 .
$$

Thus since $(I-z) \bar{a}^{*} z \bar{a}(I-z) \neq 0$, choosing vectors in the subspace determined by $I-z$ shows that

$$
\begin{aligned}
1 & =\|z \bar{a}(I-z)+(I-z)\|^{2}=\left\|(z \bar{a}(I-z)+(I-z))^{*}(z \bar{a}(I-z)+(I-z))\right\| \\
& =\left\|(I-z) \bar{a}^{*} z \bar{a}(I-z)+(I-z)\right\|>1,
\end{aligned}
$$

a contradiction. The other cases are proved in the same manner.

It follows that

$$
J_{1}=z J_{1} z+(I-z) J_{1}(I-z)=z J_{1}
$$

and similarly, $J_{2}=(I-z) J_{2}$. Thus $J_{1} J_{2}=J_{2} J_{1}=0$, and the subalgebras $J_{k}$ are (two-sided) ideals in $A^{* *}$.

We next prove that $J$ has a contractive approximate relative identity. Since $z$ is in $\bar{J} \cong J^{* *}$, there is a net $u_{\alpha} \in J$ with $\left\|u_{\alpha}\right\| \leq 1$ which converges to $z$ in the weak* topology. It follows that if $a \in J$, then $u_{\alpha} a-a$ and $a u_{\alpha}-a$ converge 
weakly to $z a-a=a z-a=0$. Given a finite set $F=\left\{a_{1}, \ldots, a_{n}\right\} \subseteq J$, it follows that $\mathbf{0}=\left(0_{1}, \ldots, 0_{2 n}\right)$ lies in the weak closure of the set

$$
V_{F}=\left\{\left(u a_{1}-a_{1}, \ldots, u a_{n}-a_{n}, a_{1} u-a_{1}, \ldots, a_{n} u-a_{n}\right): u \in J_{0}\right\} \subseteq A^{2 n},
$$

where $J_{0}$ is the closed unit ball of $J$, and we give $A^{2 n}$ the sup norm. Since $V_{F}$ is convex, $\mathbf{0}$ also lies in its norm closure. We let $\mathscr{F}$ denote the finite subsets $F$ of $J$, and we define $\Lambda=\mathscr{F} \times \mathbf{N}$. The latter is a directed set under the ordering $(F, p) \preceq\left(F^{\prime}, p^{\prime}\right)$ if $F \subseteq F^{\prime}$ and $p \leq p^{\prime}$. Given $F=\left\{a_{1}, \ldots, a_{n}\right\}$ and $p \in \mathbf{N}$, it follows from our earlier remarks that $V_{F} \cap B(1 / p) \neq \varnothing$, where $B(\varepsilon)$ is the $\varepsilon$ ball about $\mathbf{0}$ in $A^{2 n}$. Thus given $\lambda=(F, p)$, we may chose $u_{\lambda} \in J_{0}$ with $\left\|u_{\lambda} a_{k}-a_{k}\right\| \leq 1 / p$ and $\left\|a_{k} u_{\lambda}-a_{k}\right\| \leq 1 / p$ for $k=1, \ldots, n$. The net $\left\{u_{\lambda}\right\}_{\lambda \in \Lambda}$ is the desired approximate identity for $J$.

Now let us assume $J$ is an ideal in $A$ with an approximate identity $\left\{u_{\nu}\right\}_{\nu \in \Lambda}$. It is easy to see from (2.4) that $\bar{J}$ is an ideal in $A^{* *}$. Passing to a subset, we may assume that $u_{\nu}$ converges in the weak* topology to an element $z \in \bar{J}$, with $\|z\| \leq 1$. Then given $a \in J$, we have that $a u_{\nu}-a$ and $u_{\nu} a-a$ converge to $a z-a=0$ and $z a-a=0$. Given $\bar{a} \in \bar{J}$, we select a net $a_{\nu} \in J$ converging in the weak* topology to $\bar{a}$. Using the fact that the left and right Arens' multiplications coincide, we conclude that

$$
\bar{a} z-\bar{a}=\lim _{\mu} \lim _{\nu} a_{\mu} v_{\nu}-a_{\mu}=0,
$$

and

$$
z \bar{a}-\bar{a}=\lim _{\mu} \lim _{\nu} v_{\nu} a_{\mu}-a_{\mu}=0,
$$

i.e., $z$ is a relative identity for the ideal $\bar{J}$. It follows that $z$ is central in $A^{* *}$ since given $\bar{a} \in A^{* *}$, we have that $\bar{a} z, z \bar{a} \in \bar{J}$, and thus $\bar{a} z=z, z \bar{a} \in \bar{J}$, and thus $\bar{a} z=z \bar{a} z=z \bar{a}$. Since $z^{2}=z$ and $\|z\| \leq 1, z$ is a selfadjoint projection. This implies that $z$ is a central projection in the $C^{*}$-algebra $C^{*}\left(A^{* *}\right)$ generated by $A^{* *}$. Thus

$$
C^{*}\left(A^{* *}\right)=C^{*}\left(A^{* *}\right) z+C^{*}\left(A^{* *}\right)(I-z)
$$

is an $L^{\infty}$ splitting, and the same applies to $A^{* *}=A^{* *} z+A^{* *}(I-z)$. We conclude that $J$ is an $M$-ideal in $A$.

It is a simple matter to construct closed algebraic ideals which are not $M$ ideals. Letting

$$
A_{1}=\left\{\left[\begin{array}{ll}
a & b \\
0 & a
\end{array}\right]\right\} \subseteq \mathbf{M}_{2}(\mathbf{C}),
$$

$A_{2}$ is a commutative operator algebra, but the nilpotent ideal

$$
J=\left\{\left[\begin{array}{ll}
0 & b \\
0 & 0
\end{array}\right]\right\}
$$

does not have an approximate identity. 


\section{ISOMORPHISMS AND TENSOR PRODUCTS}

The following consequence of Hamana's theory of injective envelopes [12] is well known to specialists:

Proposition 3.1. A unital complete isometry of unital operator algebras is an isomorphism.

Proof. Let us suppose that $A \subseteq \mathscr{B}(H), B \subseteq \mathscr{B}(K)$ are unital operator algebras and that $\varphi: A \rightarrow B$ is a unital complete isometry. From [3, Prop. 1.2.8], $\varphi$ has a unique extension to a unital completely positive map $\varphi_{1}: A+A^{*} \rightarrow B+B^{*}$ determined by $\varphi_{1}\left(a+b^{*}\right)=\varphi(a)+\varphi(b)^{*}$. It follows from the argument given there that $\varphi_{1}$ is completely isometric and thus a complete order isomorphism (as was noted by Hamana [12, p. 781]). We let $(R, \tau)$ denote an injective envelope of $A+A^{*}$ [12]. This consists of an injective $C^{*}$-algebra $R$ and a unital complete order injection $\tau: A+A^{*} \rightarrow R$ such that if $\theta: R \rightarrow R$ is a completely positive map which extends $\tau$, then $\theta$ is the identity map. In fact we may take $R$ to be the image of a "minimal" conditional expectation $\Phi: \mathscr{B}(H) \rightarrow \mathscr{B}(H)$. Although $R$ is not generally a subalgebra of $\mathscr{B}(H)$, it has an associative product given by $r \circ s=\Phi(r s)$. In particular we see that since $A$ is contained in the range of $\Phi$, it is a subalgebra of $R$. We similarly let $(S, \kappa)$ be an injective envelope of $B+B^{*}$, with $B$ a subalgebra of S. Owing to the injectivity of $R$, we may extend $\varphi_{1}$ and $\varphi_{1}^{-1}$ to unital completely positive maps $\alpha: R \rightarrow S$ and $\beta: S \rightarrow R$. Then since $\beta \circ \alpha$ coincides with the identity on $A+A^{*}$ it must coincide with identity on $R+R^{*}$, and the same applies to $\alpha \circ \beta$, i.e., $\alpha$ and $\beta$ are inverse maps. It follows that $\alpha$ is a unital complete order isomorphism of $R$ onto $S$. Two applications of the Kadison-Schwarz inequality for completely positive maps shows that $\alpha\left(x^{*} x\right)=\alpha(x)^{*} \alpha(x)$, and then Choi's multiplicative domain technique may be used to show that $\alpha$ is a *-isomorphism (see $[6, \S 3])$. We conclude that its restriction $\varphi$ to $A$ is an isomorphism of operator algebras.

Turning to some examples, consider the commutative operator algebra

$$
A_{3}=\left\{\left[\begin{array}{lll}
a & b & c \\
0 & a & 0 \\
0 & 0 & a
\end{array}\right]\right\}=\operatorname{span}\left\{I, e_{12}, e_{13}\right\} \subseteq \mathbf{M}_{3} \text {. }
$$

The transpose map $\tau: A_{3} \rightarrow \mathbf{M}_{3}: x \rightarrow x^{t}$ is an isometric isomorphism. Letting

$$
x=\left[\begin{array}{ll}
e_{12} & 0 \\
e_{13} & 0
\end{array}\right] \text {, }
$$

we have $\|x\|=1$; but since

$$
\tau_{2}(x)=\left[\begin{array}{ll}
e_{21} & 0 \\
e_{31} & 0
\end{array}\right]
$$

$\left\|\tau_{2}(x)\right\|=\sqrt{2}$, i.e., $\tau_{2}$ is not contractive, and $\tau$ is not completely isometric. 
This example can be modified to give an example of an isometric representation $\pi$ which is not even completely bounded. Letting

$$
A_{n}=\operatorname{span}\left\{I, e_{12}, \ldots, e_{1 n}\right\} \subseteq \mathbf{M}_{n},
$$

and $\tau_{n}$ be the transpose representation of $A_{n}$, we simply let

$$
\pi=\bigoplus \tau_{n}: \bigoplus A_{n} \rightarrow \bigoplus \mathbf{M}_{n} .
$$

Given operator algebras $B_{k} \subseteq \mathscr{B}\left(H_{k}\right) \quad(k=1,2)$, the spatial matricial norms on the algebraic tensor product $B_{1} \otimes B_{2}$ are those determined by the embedding $B_{1} \otimes B_{2} \hookrightarrow \mathscr{B}\left(H_{1} \otimes H_{2}\right)$. Paulsen and Power showed in [13] that this norm does not depend on the completely isometric representations $B_{k} \hookrightarrow$ $\mathscr{B}\left(H_{k}\right)$. Essentially following these authors, we say that an operator norm structure on $B_{1} \otimes B_{2}$ is a cross norm matricial structure if one has that for $b_{k} \in B_{k}(k=1,2)$ that $\left\|b_{1} \otimes b_{2}\right\|=\left\|b_{1}\right\|\left\|b_{2}\right\|$, and the mappings $B_{1} \rightarrow B_{1} \otimes \mathbf{C} 1$, $B_{2} \rightarrow \mathbf{C} 1 \otimes B_{2}$ are completely isometric. In the next example we show that the spatial matricial norm need not be minimal among these norms.

We define a commutative operator algebra $B \subseteq \mathbf{M}_{6}$ by letting $B=\{x \oplus$ $\left.x^{t}: x \in A_{3}\right\}$. We wish to show that the spatial norm on $B \otimes B$, i.e., that determined by the injection $B \otimes B \hookrightarrow \mathbf{M}_{36}$ need not be minimal in the above sense. The map

$$
\theta: B \otimes B \rightarrow \mathbf{M}_{9} \oplus \mathbf{M}_{9}:\left(x \oplus x^{t}\right) \otimes\left(y \oplus y^{t}\right) \rightarrow\left(x \otimes y^{t}\right) \oplus\left(x^{t} \otimes y\right)
$$

is an algebraic isomorphism, and we use it to define a matricial norm structure $\left\{\|\mid \cdot\|_{n}: n \in \mathbf{N}\right\}$ on $B \otimes B$. It is a simple matter to verify that the latter is a cross matricial norm structure. The maps $\psi_{1}: x \oplus x^{t} \rightarrow x$ and $\psi_{2}: x \oplus x^{t} \rightarrow$ $x^{t}$ are completely contractive. It follows from the general properties of the spatial tensor product that $\psi_{k}(k=1,2)$ are completely contractive, and thus $\psi_{1} \otimes \psi_{2}, \psi_{2} \otimes \psi_{1}$, and $\theta=\left(\psi_{1} \otimes \psi_{2}\right) \oplus\left(\psi_{2} \otimes \psi_{1}\right)$ are completely contractive. On the other hand, letting

$$
u=\left(e_{12} \oplus e_{12}^{t}\right) \otimes\left(e_{12} \oplus e_{12}^{t}\right)+\left(e_{13} \oplus e_{13}^{t}\right) \otimes\left(e_{13} \oplus e_{13}^{t}\right) \in B \otimes B,
$$

we have that $\|u\|=\sqrt{2}$, whereas $\|u\|=\|\theta(u)\|=1$. These norms are easily calculated by considering elements of $B \otimes B \subseteq B \otimes M_{6}$ as $6 \times 6$ matrices over $B$, and elements of $\mathbf{M}_{9} \oplus \mathbf{M}_{9}$ as direct sums of $3 \times 3$ matrices over $\mathbf{M}_{3}$. It should be noted that in contrast to

$$
u=\left[\begin{array}{cccccc}
0 & e_{12} \oplus e_{21} & e_{13} \oplus e_{31} & 0 & 0 & 0 \\
0 & 0 & 0 & 0 & 0 & 0 \\
0 & 0 & 0 & 0 & 0 & 0 \\
0 & 0 & 0 & 0 & 0 & 0 \\
0 & 0 & 0 & e_{12} \oplus e_{21} & 0 & 0 \\
0 & 0 & 0 & e_{13} \oplus e_{31} & 0 & 0
\end{array}\right] .
$$


in which two " 1 's" can occur in a row or a column of the corresponding $36 \times 36$ matrix of scalars, rows, and columns of the $9 \times 9+9 \times 9$ matrix

$$
\begin{aligned}
\theta(u) & =\left(e_{12} \otimes e_{12}^{t}\right) \oplus\left(e_{12}^{t} \otimes e_{12}\right)+\left(e_{13} \otimes e_{13}^{t}\right) \oplus\left(e_{13}^{t} \otimes e_{13}\right) \\
& =\left[\begin{array}{ccc}
0 & e_{21} & e_{31} \\
0 & 0 & 0 \\
0 & 0 & 0
\end{array}\right] \oplus\left[\begin{array}{ccc}
0 & 0 & 0 \\
e_{12} & 0 & 0 \\
e_{13} & 0 & 0
\end{array}\right] .
\end{aligned}
$$

contain at most one nonzero entry.

\section{REFERENCES}

1. E. Alfsen and E. Effros, Structure in real Banach spaces, Ann. of Math. 96 (1972), 98-173.

2. R. Arens, The adjoint of a bilinear operations, Proc. Amer. Math. Soc. 2 (1951), 839-848.

3. W. Arveson, Subalgebras of $C^{*}$-algebras, Acta Math. 123 (1969), 141-224.

4. D. Blecher, Z. Ruan, and A. Sinclair, A characterization of operator algebras, J. Funct. Anal. (to appear).

5. F. Bonsall and J. Duncan, Numerical ranges of operators on normed spaces and elements of normed algebras, Cambridge Univ. Press, 1971.

6. M.-D. Choi, $A$ Schwarz inequality for positive linear maps on $C^{*}$-algebras, Illinois J. Math. 18 (1974), 565-574.

7. P. Civin and B. Yood, The second conjugate space of a Banach algebra as an algebra, Pacific J. Math. 11 (1961), 847-870.

8. M. Cunningham, E. Effros, and N. Roy, M-structure in dual Banach spaces, Israel J. Math. 14 (1973), 304-309.

9. J. Dixmier, Les $C^{*}$-algèbres et leurs representations, Gauthier-Villars, Paris 1964.

10. E. Effros, Advances in quantized functional analysis, Proc. I.C.M. Berkeley, 1986.

11. T. Gamelin, C. Marshall, R. Younis, and W. Zame, Function Theory and M-ideals, Ark. Mat. 23 (1985), 261-279.

12. M. Hamana, Injective envelopes of operator systems, Publ. Res. Inst. Math. Sci. Kyoto Univ. 15 (1979), 773-785.

13. V. Paulsen and S. Power, Tensor products of non-selfadjoint operator algebras, Rocky Mountain J. Math. (to appear).

14. R. Smith, An addendum to "M-ideal structure in Banach algebras," J. Funct. Anal. 32 (1979), 269-271.

15. R. Smith and J. Ward, M-ideal structure in Banach algebras, J. Funct. Anal. 27 (1978), 337-349.

Department of Mathematics, University of California at Los Angeles, Los Angeles, CALIFORNIA 90024

Department of Mathematics, University of Illinois, Urbana, Illinois 61801 